\title{
Nano-enabled orientation alignment via extreme shear strains
}

\author{
I. J. Beyerlein ${ }^{\text {1a }}$, J. S. Carpenter ${ }^{2}$, A. Hunter ${ }^{3}$, L. S. Toth ${ }^{4}$, W. Skrotzki ${ }^{5}$ \\ ${ }^{1}$ Theoretical Division, Los Alamos National Laboratory, Los Alamos, NM 87545, USA. \\ ${ }^{2}$ Materials Science and Technology Division, Los Alamos National Laboratory, Los Alamos, \\ NM 87545, USA. \\ ${ }^{3}$ X Computational Division, Los Alamos National Laboratory, Los Alamos, NM 87545, USA. \\ ${ }^{4}$ Laboratoire d'Etude des Microstructures et de Mécanique des Matériaux (LEM3), \\ Université de Lorraine - Metz, F-57045 METZ Cedex 01, France \\ ${ }^{5}$ Institut für Strukturphysik, Technische Universität Dresden, D-01062 Dresden, Germany
}

\begin{abstract}
It was recently shown that the crystals in an already nanocrystalline alloy $(\mathrm{Pd}-10 \% \mathrm{Au})$ became highly oriented under extreme shearing. No such self-ordering is seen in the coarse-grained counterpart under the same conditions. Here we use phase field dislocation dynamics and polycrystalline plasticity modeling to explore the possible causes that enabled this unusual crystallographic alignment. The results suggest that full-dislocation slip and preservation of grain boundary misorientation with at least one neighbor grain can cause the highly oriented texture observed experimentally.
\end{abstract}

Keywords: texture, nanocrystals, severe plastic deformation, palladium, crystal plasticity

\section{Introduction:}

Many elastic, plastic, optical and electrical properties of metals can be greatly improved by simultaneously refining the grain size to nanoscale dimensions and creating highly oriented crystals [1-4]. Nanostructured metals with preferred lattice orientations (texture) can be produced via any near-equilibrium fabrication processes, such as phase transformations, solidification, or magnetron sputtering [1, 5-8]. Far-from-equilibrium processes, such as large strain plastic deformation, can also produce nanostructured metals with preferred crystal orientations, but these are comparatively more dispersed [9-11]. Unlike the former, the latter, metal-working techniques make material in quantities suitable for structural application. Therefore, there is great interest in finding a physical way to attain a more highly oriented "bulk" nanostructured metal.

A basic deformation mode commonly used in bulk processing techniques for nanostructuring metals is simple shearing. After large simple shear strains, lattice

a Corresponding author: Irene@lanl.gov, 505-665-2231 

in face centered cubic, fcc, metals) $[12,13]$. Any attempts to sharpen the texture by changing material details usually only alters the relative fractions of particular orientations along the $\mathrm{A}$ and $\mathrm{B}$ fibers, which are conventionally labeled $B / \bar{B}, A / \bar{A}, A_{1}{ }^{*}, A_{2}{ }^{*}$, and $\mathrm{C}[10$, 14] (see Fig. 1 caption for the corresponding crystal orientations). In other words, plastic deformation still leaves the lattice orientations distributed.

A recent study of the texture evolution in an initially nanocrystalline $(\sim 15 \mathrm{~nm}) \mathrm{Pd}-10 \% \mathrm{Au}$ alloy subjected to HPT countered this thought [15]. Post-deformation microstructure and texture analyses showed that after stress-induced grain growth above $\sim 20 \mathrm{~nm}$, the texture became, both unusual and highly oriented with predominant intensity at the $B / \bar{B}$ orientations. Unlike the starting coarse-grained (CG) materials used in previous severe simple shear studies, this starting material was already nanostructured.

This result was puzzling because predominance of the $B / \bar{B}$ orientation in shear is a common signature of partial-dislocation mediated (PDM) slip yet PDM slip is not expected for the Pd-10\%Au alloy. Pd-10\%Au has a relatively high SFE (> $150 \mathrm{~mJ} / \mathrm{m}^{2}$ ) [16] and PDM slip is associated with low stacking fault energy (SFE) metals (e.g., $<40 \mathrm{~mJ} / \mathrm{m}^{2}$ ). As anticipated, characterization of the Pd-10\%Au sample after HPT via transmission electron microscopy (TEM) and X-ray line profile analysis did not reveal a substantial fraction of stacking faults $(<2 \%)[15,17,18]$ that are usually associated with frequent glide of partial dislocations. Therefore, persistence of the $B / \bar{B}$ orientations in the high SFE nanocrystalline $\mathrm{Pd}-10 \% \mathrm{Au}$ may be a consequence of an as yet undiscovered variable that operates in nanostructured but not in CG metals.

In this work using phase field and crystal plasticity methods, we demonstrate that stable, predominant $B / \bar{B}$ orientations can arise due to a combination of full-dislocation mediated (FDM) plasticity and preservation of grain boundary misorientation with at least one grain neighbor. The greater implication is that stable orientations in severe plastic deformation (SPD) may depend on whether the starting crystals are initially nano-grained or coarsegrained, suggesting a non-conventional way to control texture.

Nanocrystalline $\mathrm{Pd}-10 \% \mathrm{Au}$ was fabricated by inert gas condensation (IGC) and subsequently severely strained via HPT [see supplement Methods]. IGC creates a modellike nanocrystalline system with crystallographically randomly oriented, nearly equiaxed grains, with an average size of $\sim 15 \mathrm{~nm}$. HPT permits application of controllable as well as radically large strain levels of simple shearing [19].

To measure the texture evolution, micro X-ray diffraction was used on disks subjected to different strain levels [see supplement Methods]. HPT creates strain and stress gradients and thus spatially resolved X-ray diffraction is necessary to measure textures in HPT samples. Texture is generally presented as a 3D orientation distribution function (ODF) of intensities in which a crystal orientation is represented by three Euler angles $\left(\varphi_{1}, \Phi, \varphi_{2}\right)[14$, $20]$. To facilitate study of their evolution, we examine the $2 \mathrm{D}$ section $\left(\varphi_{1}, \Phi, \varphi_{2}=45^{\circ}\right)$ of the 3D ODF. As shown by the key in Fig. 1c, this section exposes the entire $\boldsymbol{B}$ fiber and all major 
orientations of the $\boldsymbol{A}$ fiber in separate locations $\left(A / \bar{A}, A_{1}{ }^{*}, A_{2}{ }^{*}, B / \bar{B}\right.$, and C), conventionally seen in simple shear textures. Using this $45^{\circ}$ section, Fig. 1a shows the measured evolution of texture at different strain levels over the period of large strain deformation (up to 8.0). Other regions of the disk exhibited the same texture [15].

In Fig. 1a, we see that with increasing applied strain, the intensity of the $B / \bar{B}$ orientations increased, signifying that the other conventional shear orientations are unstable and reoriented to the $B / \bar{B}$ orientation. The preference for the $B / \bar{B}$ orientations is established relatively early in the deformation at a shear strain of about 1 , corresponding to a grain size $>20 \mathrm{~nm}^{\mathrm{b}}$ [15]. While further deformation above $\sim 1$ caused some texture variations with shear strain, the predominance of the $B / \bar{B}$ does not change up to a shear strain of 16 . Development and persistence of a sharp texture is a strong indication that slip occurred inside the grains. Grain boundary sliding was not predominant. The question that now remains is what type of slip is active, PDM or FDM slip?

Previously, post-mortem TEM was employed to provide some indirect evidence of the active deformation mechanisms $[17,18]$. TEM revealed that a large fraction of the grain interiors were clear of stacking faults and dislocation debris. Lack of profuse stacking faults is usually taken to mean that PDM slip was not the predominant mechanism.

To date, the prevalent mechanisms cannot be seen experimentally in action during deformation. Therefore, it is not obvious how the density of stacking faults post mortem are related to the amount of partial slip during deformation. Toward some insight, we mention recent MD simulations of multi-crystals of $\mathrm{Cu}$ and $\mathrm{Pd}$ with $40 \mathrm{~nm}$-sized columnar grains deformed at high rates $\left(10^{8} / \mathrm{s}\right)$ [21]. It was found that in $\mathrm{Pd}$, full dislocations primarily carried the deformation and residual stacking faults were infrequent. In contrast, in $\mathrm{Cu}$, partial dislocations accommodated most of the deformation and many remnant stacking faults were left behind. According to these dynamic simulations, materials deforming via PDM slip, like nanocrystalline $\mathrm{Cu}$, would have developed numerous stacking faults within the grains after deformation. These simulations also support the notion that a lack of SFs, in materials like Pd, correlates to slip dominated by full dislocation motion.

For a given metal, transitions from FDM slip to PDM slip are thought to depend on grain size, strain rate, and fault energy landscape of the material (the $\gamma$-surface) [22-24]. Compared to the foregoing MD simulations, our grains are smaller (20-25 nm vs. $40 \mathrm{~nm}$ ) and our strain rates lower $\left(10^{1} / \mathrm{s}\right.$ vs $\left.10^{9} / \mathrm{s}\right)$. Also, the present material is an alloy, not pure Pd. To study the FDM-to-PDM cross-over in conditions closer to the present experiment, we employ a recently developed atomistically informed phase field dislocation dynamics (PFDD) technique [25-27]. In PFDD, the dynamics are driven by energy minimization according to Ginzburg-Landau [28], while the governing energy functional includes the full 3D $\gamma$-surface calculated from atomic-scale simulations [see supplement Methods] (Fig. 2a). Prior work has demonstrated the ability of this approach to nucleate and form partial dislocations and deformation twins as a function of nanograin size [26, 29].

\footnotetext{
$\mathrm{b}$ This suggests that grain boundary-mediated deformation dominated at grain sizes lower than $20 \mathrm{~nm}$.
} 
In the present PFDD simulations, the strain rate and room temperature conditions are consistent with experiment and the $\gamma$-surface calculated from MD for Pd-10\%Au was incorporated [16]. To examine Shockley partial emission from GBs, we set up a 3D cubical nanocrystal with side length $\mathrm{D}$ and inserted an atomic-scale step in one of the six GBs that protrudes on a single $\{111\}$ plane (Fig. 2b). A stress is then applied at a threshold value, just sufficient to emit a leading partial, and held constant thereafter. The grain boundaries are not permitted to slide. These simulations can indicate whether PDM or FDM slip is the energetically favorable path.

We first studied nanograin sizes greater than $20 \mathrm{~nm}$, where grain boundary sliding is presumed not to dominate. Figure $2 \mathrm{c}$ shows that for $\mathrm{D}=35.6 \mathrm{~nm}$, nucleation of a leading partial $a / 6[11 \overline{2}]$ is followed by nucleation of a correcting trailing partial $a / 6[2 \overline{1} \overline{1}]$ to form a full dislocation. A stacking fault is not stable in a grain of this size. Thus, for Pd-10\%Au, these calculations imply that PDM slip is not predominant in the grain size/temperature regime of the experiment in which the $B / \bar{B}$ orientations prevail.

As a means of validating the PFDD simulations, we repeated these calculations for $\mathrm{Cu}, \mathrm{a}$ more widely studied fcc metal, with a similar grain size $\mathrm{D}=32.9 \mathrm{~nm}$ (Fig. 2e). For $\mathrm{Cu}$, a stable stacking fault was able to extend fully across the grain without subsequent nucleation of a trail (Fig. 2e). Therefore at room temperature, a stacking fault is stable for a $\mathrm{Cu}$ grain this size. In fact, this model predicts a stable fault for sizes at least as large as 130 $\mathrm{nm}$ [30]. This PFDD prediction is consistent with MD simulation and room temperature experiments on nanostructured $\mathrm{Cu}$, where stacking fault and twin formation are observed in crystals up to $150 \mathrm{~nm}$ in size [22, 31-35]. Also, previously PDM was shown to be the relevant mechanism in the formation of a brass-type texture in large strain rolling of ultrafined grained copper $[31,36]$.

In light of these results, we pursued using the PFDD model to determine the critical grain size at which partial slip is preferred, in the event that grain boundary sliding does not occur. We repeated the calculation for $\mathrm{Pd}-10 \% \mathrm{Au}$ using a smaller grain size $\mathrm{D}=17.8 \mathrm{~nm}$. As before, the trailing partial is eventually emitted after the leading partial, forming a full dislocation (Fig. 2d). We continued refining the grain size in these calculations and found that the maximum grain size to stabilize a SF (at room temperature) is $\sim 4.4 \mathrm{~nm}$ (see supplement Fig. S1). Thus, the grain sizes at which finally PDM could prevail coincides with those at which grain boundary sliding dominates.

Having studied deformed single nanocrystals, we progressed to modeling the nanocrystalline aggregate in severe simple shearing. To relate texture evolution with the underlying deformation mechanisms, we employ a mean-field crystal plasticity model, called visco-plastic self-consistent (VPSC) $[37,38]$. Several works have demonstrated that VPSC is capable of predicting textures in crystalline materials to extreme plastic strains in the range of $\sim 10-20$, similar to the levels tested here [10]. More information on VPSC is given in the supplement. 
Based on results from MD [21] and PFDD simulations [Fig. 2], we permit the individual crystals in the VPSC model to only use combinations of the 12 full-dislocation slip systems $\{111\}<110>$ to accommodate deformation. As part of the model input, the glide resistance (hardening law) for these systems needs to be defined. Nanocrystals store little to no dislocations within their interiors $[9,11,15,39,40]$ and, hence, dislocations emitted from one grain boundary glide without hindrance to another grain boundary where they are absorbed [23, 41-43]. Consequently, unlike CG metals, conventional 'strain hardening' is suppressed in nano-grains. To reflect this for nanocrystals, we assume that the glide resistance remains constant throughout deformation.

As another nanoscale effect, we invoked in the model a strong grain-neighbor constraint in which the misorientation between neighboring grains is preserved during deformation. Plastic deformation studies in other initially nanostructured metals, also produced by a near-equilibrium thermodynamic process, have reported evidence of co-rotation [31, 44]. For example, maintenance of the Kurdjumov-Sachs orientation relationship was used to explain unusual texture development seen when a nanolayered $\mathrm{Cu}-\mathrm{Nb}$ film (magnetron sputtering) was rolled to large strains (with crystal sizes refined to 30-40 nm) [44]. As another example, preservation of the cube-on-cube orientation relationship in a rolled dual-phase $\mathrm{Cu}$-Ag nanolamellar cast eutectic was able to clarify the usual bulk texture development and local emission of twins from interfaces [31]. On this basis, we propose that the present experiment, severe shearing of an already nanocrystalline $\mathrm{Pd}-10 \% \mathrm{Au}$ fabricated by IGC, could be another manifestation of the same phenomenon. To test this notion, we used a co-rotation model introduced previously into the VPSC simulations, wherein every grain is assigned one neighbor for whom its misorientation during the entire deformation is preserved [45] (see supplement section on Methods and Fig. 4 for more details).

Fig. 1 compares the calculated textures from the polycrystal model described above with the measurement. A direct comparison of the $\mathbf{A}$ and $\mathbf{B}$ fibers can be found in supplement Fig. S2. In Fig. 1, we quickly see the predominance of the $B / \bar{B}$ orientations emerging in the calculation, in agreement with the measurement. Minor $A_{2}{ }^{*}$ and $C$ orientations not seen in the experiment are also predicted but at much lower intensity. Notably, the calculated texture agrees well with respect to the evolution with strain and intensity starting from a shear strain of 1.0 . Even the $10^{\circ}$ shift in the experimental peak from the ideal $B$ orientation (in the anti-shear sense) is captured.

We note that the intensity of the texture is, however, lower than that of the experiment. This is a consequence of grain-grain co-rotation, the maintenance of misorientation between grain pairs. As described in the supplement and in Fig. 4, the VPSC model calculates an individual lattice spin for each grain and then subsequently a corrective rotation is added such that a constant disorientation angle is maintained [45]. Ideal orientations can still prevail but are more likely to be weak because a grain that is less ideally oriented could make it difficult for its neighboring grain to reach an ideal position. In other words, the less stable grain of the pair would govern the response, which is also seen in co-deforming crystals in rolling [46]. 

nanocrystalline metal, defects are stored inside the grain and within the grain boundaries [19, 47-49]. In addition, intra-granular misorientations can develop and extrinsic dislocations can be stored within the grain boundaries. These mechanisms can accommodate any changes in grain boundary misorientation during deformation. Modeling texture evolution in a CG material thus corresponds to repeating the VPSC calculation with $\{111\}<110>$ slip, ordinary hardening, and suppressing co-rotation between grain neighbors. Fig. 3a shows the corresponding predicted texture, which exhibits the classic shear texture, in agreement with measurements made on CG metals subjected to HPT [19]. Thus, without the constraint of co-rotation between neighbors, the sheared microstructure does not become highly oriented at $B / \bar{B}$. We conclude then that the development of the highly oriented $B / \bar{B}$ texture can arise when neighboring nanocrystals co-rotate, resisting changes in misorientation during deformation.

It may be the case that the absence of stacking faults and twin boundaries in postdeformation TEM evaluation could be a consequence of "full-grain twinning" rather than PDM or FDM. In other words, the entire grain orients to a twin orientation, so that internal parent/twin boundaries are absent. To explore this possibility, we carried out polycrystal VPSC calculations allowing grains to undergo deformation twinning on the highest driven $\{111\}<112>$ twinning variant and reorient completely to the orientation of this variant [31]. Figure $3 \mathrm{~b}$,c shows the predictions at different levels of twin volume fraction $v_{\mathrm{f}}$. Regardless of $v_{\mathrm{f}}$, it is clear that a strong $A_{1}{ }^{*}$ orientation is stabilized. As validation, this finding agrees with texture measurements for $\mathrm{Ag}$ after extreme simple shearing, a metal that is known to deformation twin easilyc [50]. However, the lack of prevailing $A_{1}{ }^{*}$ orientations in the texture measurement of the severely sheared Pd-10\%Au strongly indicates that the lack of stacking faults was not due to profuse twinning.

With the above set of calculations, we have shown that co-rotation between neighboring grains deforming by full dislocation slip can cause a predominant $B / \bar{B}$ orientations to emerge in a nanocrystalline material deformed under extreme shear strains. Prior studies have shown that it is also possible for mean-field crystal plasticity simulations with PDM slip and without the co-rotation constraint to predict a similar $B / \bar{B}$ dominant texture in agreement with experimental measurement [15]. The weak point of that simulation is that the partial dislocations were not found in the HPT deformed Pd-10\%Au alloy. Moreover the present dynamic PFDD simulations do not support the formation of partial dislocations in the Pd-10\%Au alloy. Therefore, although PDF can also explain the same texture, no evidence support PDM slip in the actual material.

Our finding leads to the question of why co-rotation prevails and how it is accommodated. We envision that for a very fine nano-grained material the preservation of grain boundary misorientation is enabled through stress-driven atomic-scale accommodating processes operating at the grain boundary (Fig. 4). In each strain increment, crystallographic slip inside the two adjoining grains creates a plastic spin $\omega^{1}$ and $\omega^{2}$, which can cause them to

\footnotetext{
c Note that $A_{1}$ * is the twinning component for positive shear while it is the $A_{2}{ }^{*}$ component for negative shear, which was the case for ECAE.
} 
deviate from their original misorientation. However, grain boundary accommodation mechanisms can enable the grains to rotate back without slip. This collaboration is reflected in the present calculations with grain neighbor co-rotation [45]. As detailed in the supplement, in the micromechanical modeling of co-rotation a corrective spin $\omega^{\text {corot }}$ is calculated for each grain pair in each increment and is added to one grain and subtracted from its neighbor (Fig. 4) so that the GB misorientation is maintained. We hesitate to comment further on the nature of these atomic mechanisms and only remark that stressdriven GB mechanisms would be needed to preserve the GB misorientation in shear.

In summary, our findings suggest that a highly oriented nanostructure can be achieved during severe plastic shearing via preservation of grain boundary misorientation. This strong boundary constraint is not likely realized in a CG metal, which conventionally exhibits a more dispersive set of orientations after deformation. This finding introduces a different role of grain boundaries in deformation and a way toward producing highly oriented nanostructured metals.

\section{Acknowledgements}

IJB and JSC would like to acknowledge support by a Laboratory Directed Research and Development program award number 20140348ER. AH would like to acknowledge support by a Laboratory Directed Research and Development program award number 20130745ECR. LST was supported by the French State program "Investment in the future" operated by the National Research Agency (ANR) ANR-11-LABX-0008-01, LabEx DAMAS.

\section{Figure Captions:}

Fig. 1. Texture evolution on Pd-10\%Au during HPT after different levels of strain (a) measured by X-ray diffraction [15] and (b) predictions from the VPSC model assuming full dislocation slip and co-rotation. (c) key identifying the locations of the primary components $A / \bar{A}: \quad\{1 \overline{1} \overline{1}\}\left\{<110>/\{\overline{1} 11\}<\overline{1} \overline{1} 0>; B / \bar{B}: \quad\{\overline{1} 12\}<110>/\{1 \overline{1} \overline{2}\}<\overline{1} \overline{1} 0>; A_{1} *\right.$ : $\{\overline{1} \overline{1} 1\}<112>; A_{2}^{*}:\{11 \overline{1}\}<112>$; C: $\{001\}<110>$, where the orientations are indicated by the crystallographic plane and direction $\{\mathrm{SP}\}<\mathrm{SD}>$ that are parallel to the shear plane (SP) and shear direction (SD), respectively.

Fig. 2. Phase field dislocation dynamics calculations: (a) generalized stacking fault energy curves for $\mathrm{Pd}-10 \% \mathrm{Au}$ and $\mathrm{Cu},(\mathrm{b})$ model of a 3D cubical grain with a grain boundary ledge protruding on one atomic plane, and (c-e) time evolution of a stacking fault loop emitted from the grain boundary ledge under a constant stress in (c) Pd-10\%Au for $35.6 \mathrm{~nm}$ and (d) $17.8 \mathrm{~nm}$ and (e) $\mathrm{Cu}$ for $32.9 \mathrm{~nm}$. Hashed regions indicate grain boundaries. The stress to emit the leading partial from the grain boundary ledge scales with the unstable stacking fault energy $\gamma_{\text {usf }} / \mu b$, where $\mu$ is the shear modulus and $b$ is the Burgers vector length.

Fig. 3. Texture predictions from the VPSC model under large strain simple shearing (a) with ordinary hardening and full-dislocation slip, presumed to prevail in the plasticity of CG metals, and (b-c) with full-grain twinning, with 55\% and $83 \%$ twin volume fraction, 
respectively. The typical shear texture contains many of the ideal shear orientations $A / \bar{A}$, $B / \bar{B}, C$, and $A_{1}^{*}$, in agreement with experimental observations on CG metals that have been heavily sheared.

Fig. 4. Schematic of co-rotation between two neighboring grains to preserve their original misorientation. 
[1] Mara NA, Beyerlein IJ. Journal of Materials Science 2014;49:6497.

[2] Fullwood DT, Niezgoda SR, Adams BL, Kalidindi SR. Progress in Materials Science 2010;55:477.

[3] Meyers MA, Mishra A, Benson DJ. Progress in Materials Science 2006;51:427.

[4] Proust G, Kalidindi SR. Journal of the Mechanics and Physics of Solids 2006;54:1744.

[5] Beyerlein IJ, Caro A, Demkowicz MJ, Mara NA, Misra A, Uberuaga BP. Materials Today 2013;16:443.

[6] Misra A. Mechanical Behavior of Metallic Nanolaminates. In: Hill AJ, Hannink RHJ, editors. Nanostructure Control of Materials. UK: Woodhead Publishing Co., 2006. p.146.

[7] Monclus MA, Zheng SJ, Mayeur JR, Beyerlein IJ, Mara NA, Polcar T, Llorca J, MolinaAldareguia JM. Applied Physics Letters Materials 2014;1:052103.

[8] Hsiao HY, Liu CM, Lin HW, Liu TC, Lu CL, Huang YS, Chen C, Tu KN. Science 2012;336:1007.

[9] Ekiz EH, Lach TG, Averback RS, Mara NA, Beyerlein IJ, Pouryazdan M, Hahn H, Bellon P. Acta Materialia 2014;72:178.

[10] Beyerlein IJ, Toth LS. Progress in Materials Science 2009;54:427.

[11] Valiev RZ, Langdon TG. Progress in Materials Science 2006;51:881.

[12] Toth LS, Gilormini P, Jonas JJ. Acta Metallurgica 1988;36:3077.

[13] Toth LS, Neale KW, Jonas JJ. Acta Metallurgica 1989;37:2197.

[14] Kocks UF, Tome CN, Wenk H-R. Texture and Anisotropy: Preferred orientations in polycrystals and their effect on material properties. Cambridge: Cambridge University Press, 1998.

[15] Skrotzki W, Eschke A, $\sqrt{ } \sqrt{ } \geq$ ni B, Ung $\sqrt{ }^{\circ} \mathrm{r} T, \mathrm{~T} \sqrt{ } \geq$ th LS, Ivanisenko Y, Kurmanaeva L. ACTA MATERIALIA 2013;61:7271.

[16] Schafer J, Stukowski A, Albe K. Acta Materialia 2011;59:2957.

[17] Ivanisenko Y, Skrotzki W, Chulist R, Lippmann T, Kurmanaeva L. Scripta Materialia 2012;66:131.

[18] Ivanisenko Y, Skrotzki W, Chulist R, Lippmann T, Yang K, Kurmanaeva L, Fecht HJ. Journal of Materials Science 2010;45:4571.

[19] Enikeev NA, Schafler E, Zehetbauer M, Alexandrov IV, Valiev RZ. Mater Sci Forum 2008;584-586:367.

[20] Engler O, Randle V. Introduction to texture analysis. Boca Raton, FL: CRC Press, Taylor and Francis Group, 2010.

[21] Smith L, Farkas D. Philosophical Magazine 2014;94:152.

[22] Yue YH, Liu P, Deng QS, Ma E, Zhang Z, Han XD. Nano Letters 2012;12:4045.

[23] Van Swygenhoven H, Derlet PM, Froseth AG. Nature Materials 2004;3:399.

[24] Asaro RJ, Krysl P, Kad B. Philosophical Magazine Letters 2003;83:733.

[25] Hunter A, Beyerlein IJ. Materials Science and Engineering: A 2014;600:200.

[26] Hunter A, Beyerlein IJ. APL Materials 2013;1:032109.

[27] Hunter A, Beyerlein IJ, Germann TC, Koslowski M. Physical Review B 2011;84.

[28] Koslowski M. Philosophical Magazine 2007;87:1175.

[29] Hunter A, Beyerlein IJ. Applied Physics Letters 2014;104. 
[30] Hunter A, Zhang RF, Beyerlein IJ. Journal of Applied Physics 2014;115.

[31] Beyerlein IJ, Mara NA, Bhattacharyya D, Alexander DJ, Necker CT. International Journal of Plasticity 2011;27:121.

[32] McCabe RJ, Beyerlein IJ, Carpenter JS, Mara NA. Nature Communications 2014;5:3806.

[33] Beyerlein IJ, Zhang X, Misra A. Annual Review of Materials Research 2014;44:329.

[34] Zheng SJ, Beyerlein IJ, Wang J, Carpenter JS, Han WZ, Mara NA. Acta Materialia 2012;60:5858.

[35] Huang CX, Wang K, Wu SD, Zhang ZF, Li GY, Li SX. Acta Materialia 2006;54:655.

[36] Gu CF, Toth LS, Rusz S, Bova M. Scripta Materialia 2014;86:36.

[37] Lebensohn RA, Tome CN. Acta Metallurgica Et Materialia 1993;41:2611.

[38] Beyerlein IJ, McCabe RJ, Tome CN. International Journal for Multiscale Computational Engineering 2011;9:459.

[39] Beyerlein IJ, Mayeur JR, Zheng SJ, Mara NA, Wang J, Misra A. Proceedings of the National Academy of Sciences of the United States of America 2014;111:4386.

[40] Zhu YT, Liao XZ, Wu XL. Prog. Mater. Sci. 2012;57:1.

[41] Kumar KS, Suresh S, Chisholm MF, Horton JA, Wang P. Acta Materialia 2003;51:387.

[42] Kumar KS, Van Swygenhoven H, Suresh S. Acta Materialia 2003;51:5743.

[43] Wu XL, Zhu YT, Wei YG, Wei Q. Physical Review Letters 2009;103.

[44] Al-Fadhalah K, Tome CN, Beaudoin AJ, Robertson IM, Hirth JP, Misra A. Philosophical Magazine 2005;85:1419.

[45] Tome CN, Lebensohn RA, Necker CT. Metallurgical and Materials Transactions aPhysical Metallurgy and Materials Science 2002;33:2635.

[46] Mayeur JR, Beyerlein IJ, Bronkhorst CA, Mourad HM, Hansen BL. International Journal of Plasticity 2013;48:72.

[47] Valiev R. Nature Materials 2004;3:511.

[48] Xue Q, Beyerlein IJ, Alexander DJ, Gray Iii GT. ACTA MATERIALIA 2007;55:655.

[49] Nazarov AA, Romanov AE, Valiev RZ. Acta Metallurgica Et Materialia 1993;41:1033.

[50] Beyerlein IJ, Toth LS, Tome CN, Suwas S. Philosophical Magazine 2007;87:885. 
Experimental Data

Shear Strain $=1.3$

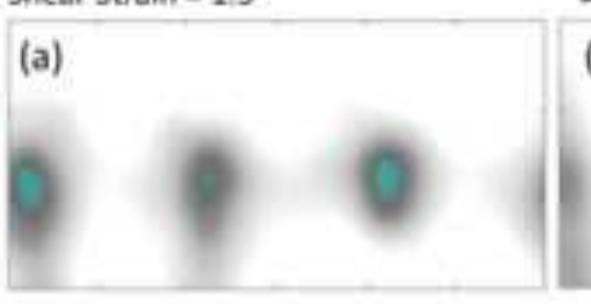

Shear 5 train $=2.8$

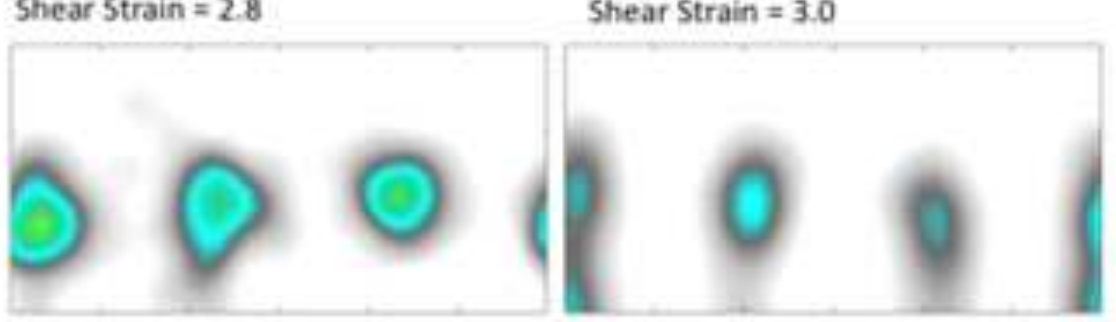

Shear Strain $=8.4$

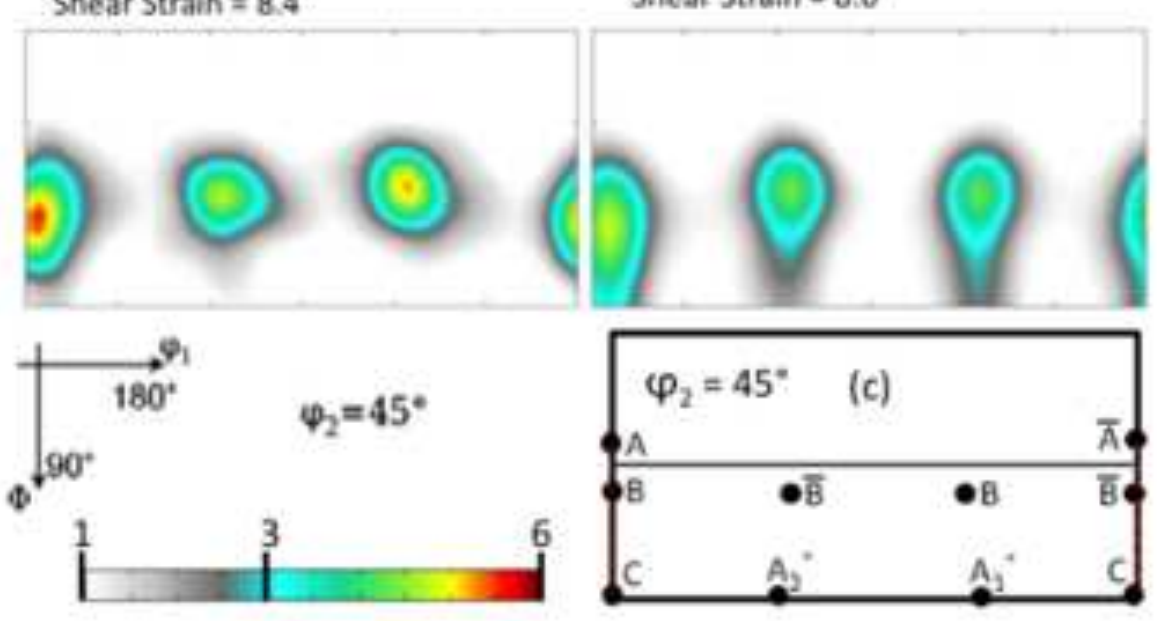

VPSC Model

Shear Strain $=1.0$

(b)

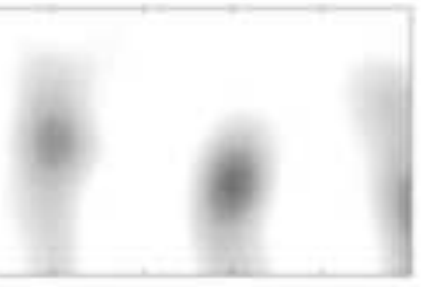

Shear Strain $=3.0$

Shear Strain $=8.0$

A 

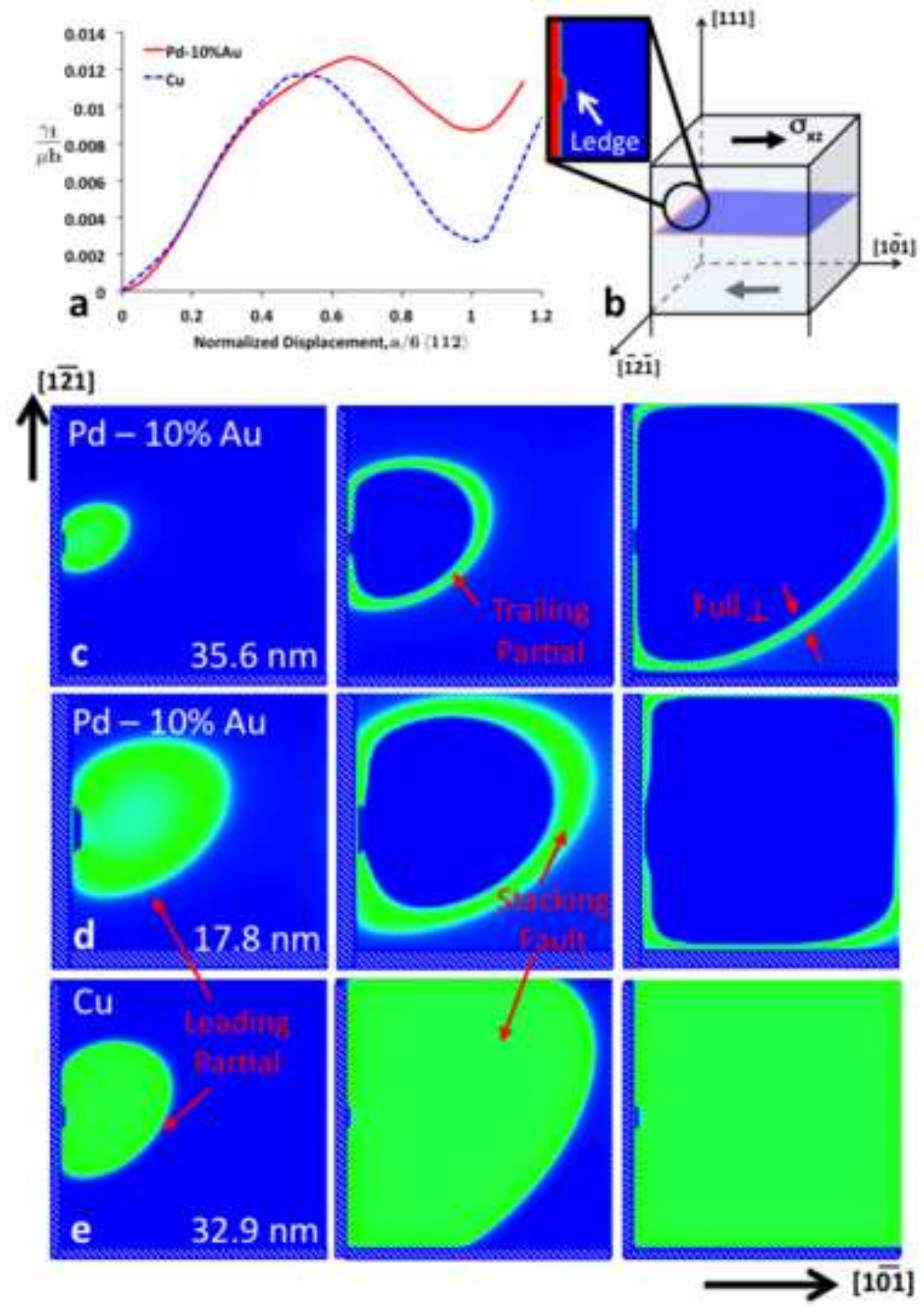


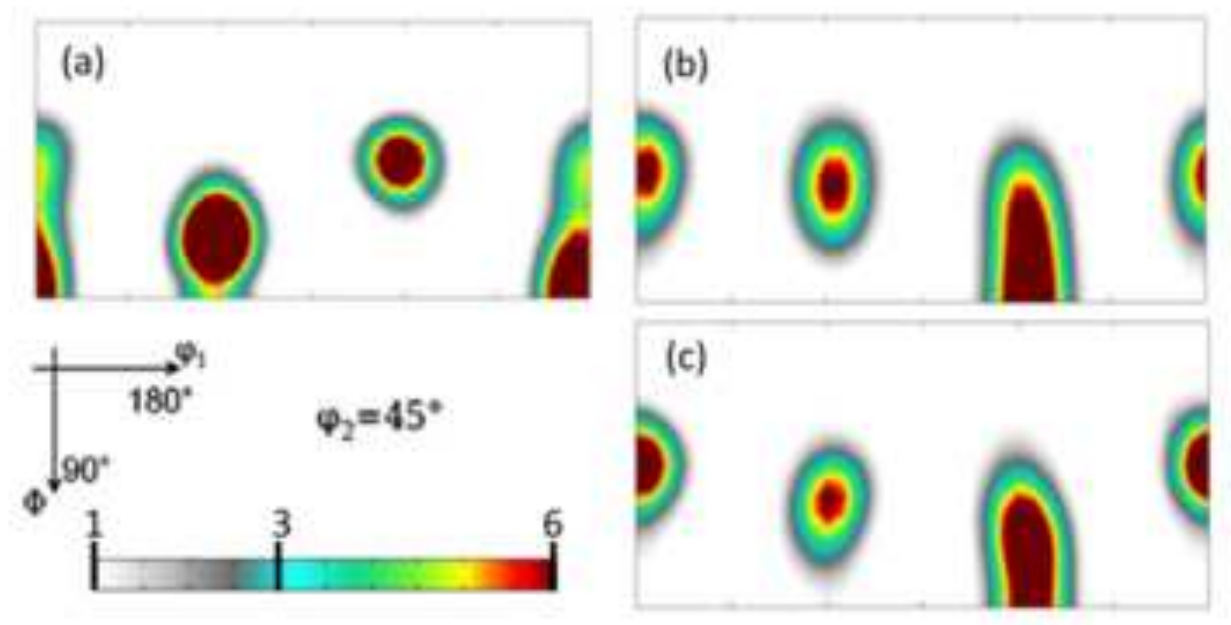




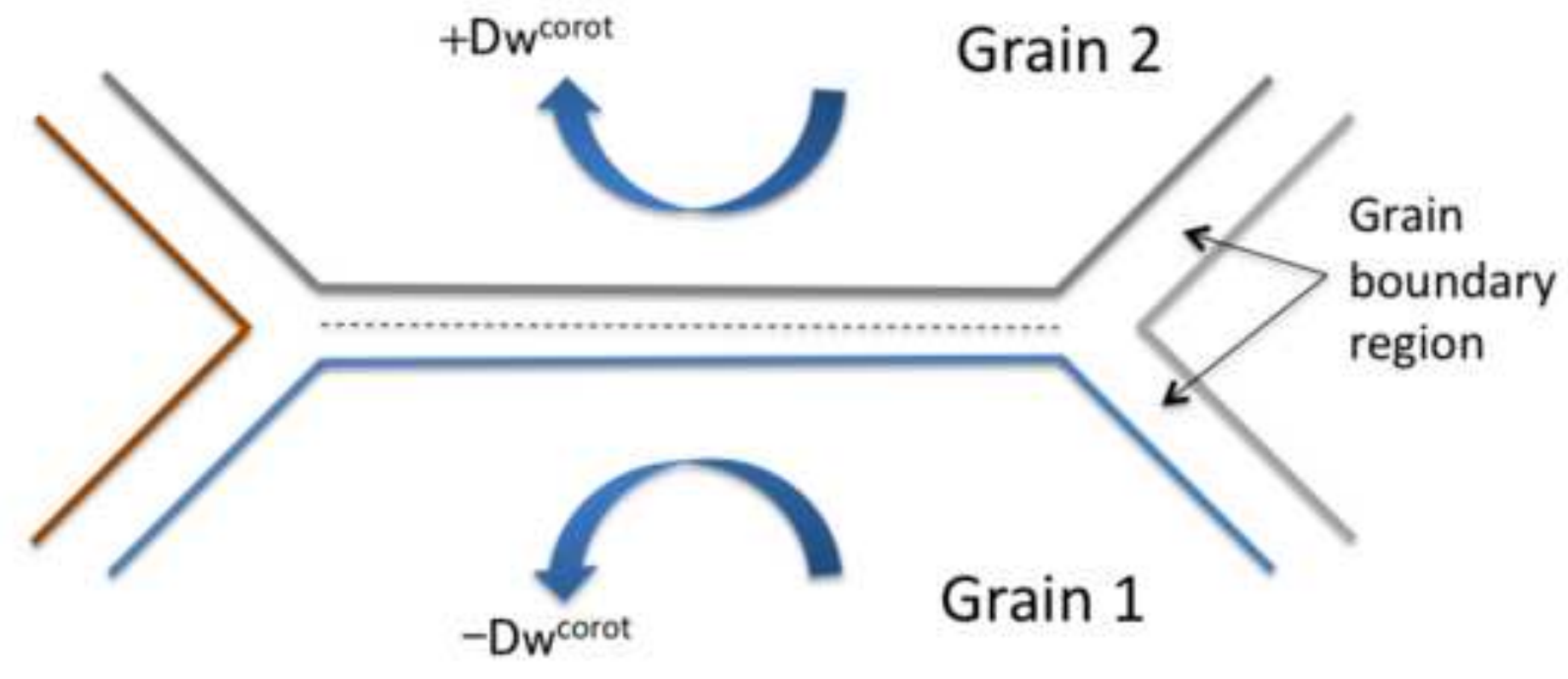

